\title{
Taking a shower with a raincoat on women's views on male condom use
}

\author{
Original \\ IhabYounis, Menha A. Ibrahim, Doaa M. Elhabak \\ Article \\ Department of Dermatology, Venereology and Andrology, Faculty of Medicine, Benha \\ University, Benha, Egypt
}

\begin{abstract}
Introduction: Condom use within marriage could be an important contraceptive strategy, but there is considerable debate about whether married couples would be willing to use condoms.

Aim of the Study: Evaluation of the effect of condom use on female sexual functions. Also, acceptability of both partners to this method will be assessed.

Materials and Methods: A total of 200 married women who experienced male condom use by their husbands were enrolled in this work. The tool used in this study was a self-report questionnaire written in English and translated into Arabic to ensure it is understood by all subjects regardless of their level of education.

Results: With condom use, desire level prior to sexual relationship was moderate in $38 \%$ of participants. Half of the respondents had moderate degree of lubrication while only $36 \%$ kept lubrication during the whole coitus on using condom. Condom had a negative effect on orgasm frequency in $28 \%$ of respondents. Most participants thought that their sexual life would be better without condom use.

Conclusion: A majority of women disliked male condoms. Unsatisfactory sex, slipped condom, vaginal dryness and pain were very common sexual side effects of condom use in our study.
\end{abstract}

Key Words: Condom, male, views, women.

Received: 13 April 2019, Accepted: 19 April 2019

Corresponding Author: Ihab Younis, MD, Department of Dermatology, Venereology and Andrology, Faculty of Medicine, Benha University, Benha, Egypt, Tel.: +201017186419, E-mail: ihabyounis@hotmail.com

ISSN: 2090-6048, March 2019, Vol. 9, No. 1

\section{INTRODUCTION}

The success of a contraceptive method depends not only on its effectiveness in preventing conception, but also on its acceptability and hence continued usage. This acceptability depends, among other factors, on the quality and quantity of the side effects ${ }^{[1]}$. For condoms in particular, embarrassment associated with purchasing them and negotiating their use has been shown to impact negatively the frequency of their use ${ }^{[2]}$.

Earlier studies support the hypothesis that persons who believe condoms 'interfere with pleasure' ${ }^{[3]}$ or who rate condom-protected sex as less enjoyable than unprotected sex may be less likely to use condoms in practice ${ }^{[4]}$.

Although public health condom promotion efforts increasingly focus on the importance of sexual pleasure, little is known about sexual event-specific factors that may increase or decrease perceptions of sexual pleasure when condoms are used ${ }^{[5]}$.
Research on condom use and affect has produced mixed results. Some research suggests that condomless sexual behavior is not associated with affect or emotion dysregulation ${ }^{[6]}$. Other research has found that individuals are less likely to use condoms on days when they experience more positive affect ${ }^{[7]}$.

\section{AIM OF THE STUDY}

The aim of the study was to identify the key factors that affect the acceptability of condom use within marriage and actual condom effect on female sexuality among a sample of Egyptian women.

\section{MATERIALS AND METHODS}

\section{Study population}

The current study was a cross-sectional study including 200 married women who experienced male condom use by their husbands. The participants were 
recruited from women attending the outpatient clinic, Benha University Hospital.

\section{Compliance with ethics guidelines}

Following the approval of Dermatology and Andrology Department and Medical Ethics Committee, Faculty of Medicine, Benha University, participants' consent was taken after explaining the purpose of the study.

\section{Data collection}

The tool used in this study was a self-report questionnaire designed by the authors guided by the Female Sexual Dysfunction Index ${ }^{[8]}$. Face to face questionnaire was not used to avoid any embarrassment and to give the participants a wide range of privacy and freedom to express themselves without any disturbance or fear.

The questionnaire included the following domains: epidemiological data, assessment of condom use modalities, and its effect on female sexual activity including arousal, desire, orgasm, lubrication, coital encounter duration, and satisfaction. Also, condom interference with sexual enjoyment of both partners was assessed.

Women with medical conditions affecting female sexuality, for example neuropsychiatric diseases and women giving a history of husband's sexual dysfunction were excluded from the study.

\section{Statistical analysis}

Data were statistically analyzed using STATA/SE version 11.0 (Stata Statistical Software; College Station, Texas, USA) for Windows. $\chi 2$ test was used to compare between two or more categorical groups (table $2 \times 2$ or more). Fisher's exact test was used to compare more than two proportions as appropriate.

$P$ value more than 0.05 was considered statistically insignificant. $P$ value less than 0.05 was considered statistically significant. $P$ value less than 0.01 or less than 0.001 was considered highly statistically significant.

\section{RESULTS}

Most of the participants (52\%) belonged to the age group of 30-39 years. Secondary school graduates comprised the most common educational level (84\%). Most participants were not working and most of them lived in urban areas (82\%) (Table 1).

Condomuse had a moderate effect on desire level in $38 \%$ of participants and affected arousal in less than half the coital encounters in $28 \%$ of the sample. Fifty percent of respondents had moderate degree of lubrication while only $36 \%$ kept lubrication during the whole coitus on using a male condom. With condom use, orgasm was almost never reached in more than a quarter of the participants $(28 \%)$, while $36 \%$ of them find slight difficulty in reaching orgasm (Table 2).

Seventy-seven percent of women found that their husbands' use of condom increases coital duration. Moderate satisfaction of coitus duration with condom use was felt by $34 \%$ of participants (Table 3 ).

Table 4 shows that $26 \%$ of participants were very satisfied with condom use. Seventy-two percent of participants think that their sexual life would be better without condom use.

Condom use decreased enjoyment in $48 \%$ of the study participants. Slipped condom was the most common complaint of wives in our study (34\%) followed by pain $(26 \%)$ and vaginal dryness $(22 \%)$. For husbands, it was different. Unsatisfactory sex was the main complaint as reported by $64 \%$ of wives followed by slipped condom (36\%) (Table 5).

Table 6 shows that condom use for oral sex is limited to age groups 20-29 years and 30-39 years. Women aged 40-49 years showed the lowest frequency of arousaland the highest percentage of inability to reach orgasm. This table also indicates that the percentage of participants who described their husbands as cooperative and understanding their role in contraception increased with advance in age. 
Table 1: Demographic data of participants $(\mathrm{n}=200)$

\begin{tabular}{lcc}
\hline Variables & $\mathrm{n}$ & $\%$ \\
\hline Age: & & 2.0 \\
$18-19$ & 4 & 30.0 \\
$20-29$ & 60 & 52.0 \\
$30-39$ & 104 & 12.0 \\
$40-49$ & 24 & 4.0 \\
$<\quad 50$ & 8 & 22.0 \\
Educational level: & & 42.0 \\
Just read and write & 44 & 36.0 \\
Secondary school & 84 & 34.0 \\
University degree & 72 & 66.0 \\
Job: & & 82.0 \\
Yes & 68 & 18.0 \\
No & 132 & 8 \\
Residence: & & \\
Urban & 164 & 36 \\
Rural & 36 & \\
\hline
\end{tabular}

Table 2: Male condom use effect on female sexual activity $(n=200)$

\begin{tabular}{|c|c|c|}
\hline Variables & $\mathrm{n}$ & $\%$ \\
\hline \multicolumn{3}{|l|}{ Desire: } \\
\hline Very high & 16 & 8.0 \\
\hline High & 4 & 2.0 \\
\hline Moderate & 76 & 38.0 \\
\hline Low & 32 & 16.0 \\
\hline Very low or none & 72 & 36.0 \\
\hline \multicolumn{3}{|l|}{ Arousal: } \\
\hline Almost always & 16 & 8.0 \\
\hline More than half the times & 32 & 16.0 \\
\hline About Half the times & 44 & 22.0 \\
\hline Less than the times & 56 & 28.0 \\
\hline Never & 52 & 26.0 \\
\hline \multicolumn{3}{|l|}{ Lubrication: } \\
\hline Very high & 12 & 6.0 \\
\hline High & 36 & 18.0 \\
\hline Moderate & 100 & 50.0 \\
\hline Low & 32 & 16.0 \\
\hline Dry & 20 & 10.0 \\
\hline \multicolumn{3}{|l|}{ Orgasm: } \\
\hline \multicolumn{3}{|l|}{ Frequency } \\
\hline Always & 20 & 10.0 \\
\hline More than half the times & 40 & 20.0 \\
\hline About half the times & 32 & 16.0 \\
\hline Less than half the times & 52 & 26.0 \\
\hline Almost never or never & 56 & 28.0 \\
\hline \multicolumn{3}{|l|}{ Ability to reach orgasm: } \\
\hline Not difficult & 40 & 20.0 \\
\hline Slightly difficult & 72 & 36.0 \\
\hline Difficult & 32 & 16.0 \\
\hline Very difficult & 40 & 20.0 \\
\hline Extremely difficult or impossible & 16 & 8.0 \\
\hline
\end{tabular}


Table 3: Effect of condom use on coital duration

\begin{tabular}{|c|c|c|}
\hline Variables & $\mathrm{n}$ & $\%$ \\
\hline \multicolumn{3}{|c|}{ Does condom affect intercourse duration $(n=200)$ : } \\
\hline Yes & 140 & 70.0 \\
\hline No & 60 & 30.0 \\
\hline \multicolumn{3}{|c|}{ If answer is yes, the duration was (no.140): } \\
\hline Longer than usual & 108 & 77.1 \\
\hline Shorter than usual & 32 & 22.9 \\
\hline \multicolumn{3}{|c|}{ Level of satisfaction by coitus duration with condom use $(n=200)$ : } \\
\hline Very satisfied & 40 & 20.0 \\
\hline Moderately satisfied & 68 & 34.0 \\
\hline Equally satisfied and dissatisfied & 40 & 20.0 \\
\hline Moderately dissatisfied & 28 & 14.0 \\
\hline Unsatisfied & 24 & 12.0 \\
\hline
\end{tabular}

Table 4: Wife's Point of view $(n=200)$

\begin{tabular}{lcr}
\hline Variables & $\mathrm{n}$ & \\
\hline Satisfaction with condom use: & 52 & 26.0 \\
$\quad$ Very satisfied & 36 & 18.0 \\
Moderately satisfied & 40 & 20.0 \\
Equally satisfied and dissatisfied & 36 & 18.0 \\
Moderately dissatisfied & 36 & 18.0 \\
Unsatisfied & 144 \\
Without condom, participant sexual life would be: & 24 \\
Better & 32 \\
Worse & 72.0 \\
No difference & 12.0 \\
\hline
\end{tabular}

Table 5: Condom problems $(n=200)$

\begin{tabular}{|c|c|c|}
\hline Variables & $\mathrm{n}$ & $\%$ \\
\hline \multicolumn{3}{|l|}{ Male condom represents: } \\
\hline Additive enjoyment tool & 72 & 36.0 \\
\hline Non-enjoyment tool & 96 & 48.0 \\
\hline Nothing & 32 & 16.0 \\
\hline \multicolumn{3}{|c|}{ Female complication with condom use:(<one answer) } \\
\hline Unplanned pregnancy & 32 & 16.0 \\
\hline Allergy & 16 & 8.0 \\
\hline Unsatisfactory sex & 28 & 14.0 \\
\hline Slipped condom & 68 & 34.0 \\
\hline Pain & 52 & 26.0 \\
\hline Dryness & 44 & 22.0 \\
\hline Others & 4 & 2.0 \\
\hline -Fear of use & 4 & 2.0 \\
\hline No problems & 32 & 16.0 \\
\hline \multicolumn{3}{|c|}{ Husband complications with condom use : (<one answer) } \\
\hline Allergy & 4 & 2.0 \\
\hline Difficulty in use & 32 & 16.0 \\
\hline Slipped condom & 72 & 36.0 \\
\hline Unsatisfactory sex & 92 & 46.0 \\
\hline Others & 4 & 2.0 \\
\hline -Fear of unplanned preg. & 3 & 1.5 \\
\hline -Fear of use & 1 & 0.5 \\
\hline No problems & 32 & 16.0 \\
\hline
\end{tabular}


Table 6: Correlation between age and condom indication, some components of FSF and husband`s point of view.

\begin{tabular}{|c|c|c|c|c|c|c|c|c|c|c|c|}
\hline \multirow{3}{*}{ Variables } & \multicolumn{11}{|c|}{ Age } \\
\hline & \multicolumn{2}{|c|}{$18-19(4)$} & \multicolumn{2}{|c|}{$20-29(60)$} & \multicolumn{2}{|c|}{$30-39(104)$} & \multicolumn{2}{|c|}{$40-49(24)$} & \multicolumn{2}{|c|}{$50+8$} & \multirow{2}{*}{$P^{*}$} \\
\hline & $\mathrm{n}$ & $\%$ & $\mathrm{n}$ & $\%$ & $\mathrm{n}$ & $\%$ & $\mathrm{n}$ & $\%$ & $\mathrm{n}$ & $\%$ & \\
\hline Condom indication & & & & & & & & & 8 & & \multirow{6}{*}{0.05} \\
\hline Contraception & 4 & 100.0 & 40 & 66.7 & 80 & 76.9 & 20 & 83.3 & 0 & 100.0 & \\
\hline Treatment of premature ejaculation & 0 & 0.0 & 0 & 0.0 & 8 & 7.7 & 0 & 0.0 & 0 & 0.0 & \\
\hline Prevention of STIs & 0 & 0.0 & 0 & 0.0 & 0 & 0.0 & 0 & 0.0 & 0 & 0.0 & \\
\hline Oral sex & 0 & 0.0 & 12 & 20.0 & 8 & 7.7 & 0 & 0.0 & 0 & 0.0 & \\
\hline Others & 0 & 0.0 & 8 & 13.3 & 8 & 7.7 & 4 & 16.7 & & 0.0 & \\
\hline \multicolumn{12}{|l|}{ Frequency of arousal } \\
\hline Almost always & 0 & 0.0 & 0 & 0.0 & 16 & 15.4 & 0 & 0.0 & 0 & 0.0 & \multirow{5}{*}{$\begin{array}{r}<0.001 \\
(\mathrm{HS})\end{array}$} \\
\hline More than half the times & 0 & 0.0 & 4 & 6.7 & 16 & 15.4 & 4 & 16.7 & 8 & 100.0 & \\
\hline About half the times & 0 & 0.0 & 20 & 33.3 & 24 & 23.1 & 0 & 0.0 & 0 & 0.0 & \\
\hline Less than half the times & 0 & 0.0 & 28 & 46.7 & 20 & 19.2 & 8 & 33.3 & 0 & 0.0 & \\
\hline Never & 4 & 100.0 & 8 & 13.3 & 28 & 26.9 & 12 & 50.0 & 0 & 0.0 & \\
\hline \multicolumn{12}{|l|}{ Frequency of reaching orgasm } \\
\hline Always & 0 & 0.0 & 0 & 0.0 & 12 & 11.5 & 0 & 0.0 & 8 & 100.0 & \multirow{5}{*}{$\begin{array}{c}<0.001 \\
\text { (HS }\end{array}$} \\
\hline More than half the times & 0 & 0.0 & 16 & 26.7 & 20 & 19.2 & 4 & 16.7 & 0 & 0.0 & \\
\hline About half the times & 0 & 0.0 & 12 & 20.0 & 16 & 15.4 & 4 & 16.7 & 0 & 0.0 & \\
\hline Less than half the times & 4 & 100.0 & 16 & 26.7 & 32 & 30.8 & 0 & 0.0 & 0 & 0.0 & \\
\hline Almost never or never & 0 & 0.0 & 16 & 26.7 & 24 & 23.1 & 16 & 66.7 & 0 & 0.0 & \\
\hline \multicolumn{12}{|l|}{ Husband Point of View } \\
\hline Cooperative and understands his role & 0 & 0.0 & 12 & 20.0 & 28 & 26.9 & 8 & 33.3 & 4 & 50.0 & \multirow{3}{*}{$\begin{array}{c}<0.001 \\
(\mathrm{HS})\end{array}$} \\
\hline Used as there is no other safe alternative & 0 & 0.0 & 28 & 46.7 & 68 & 65.4 & 8 & 33.3 & 4 & 50.0 & \\
\hline I don’t know & 4 & 100.0 & 20 & 33.3 & 8 & 7.7 & 8 & 33.3 & 0 & 0.0 & \\
\hline
\end{tabular}

*Obtained using the Fisher's Exact Test (FET)

\section{DISCUSSION}

In cultures where men do not feel responsible for family planning, discussing contraception can be a problem especially when the husband has a negative attitude toward the use of condoms. In the case of inconsistent condom use, the negative attitude of the male partner to condom use is considered as an important factor ${ }^{[9]}$.

The possible association between contraceptives (including condom) and sexual function remains controversial in the literature. It has been reported that women may experience positive effects ${ }^{[10]}$, negative effects $^{[11]}$, or no effects ${ }^{[12,13]}$ on libido and other parameters of sexual function during contraceptive use.

Some men dislike condoms because they feel that condoms either diminish sexual pleasure or are difficult to use $^{[14]}$. Some men and women develop allergic reactions to the latex ${ }^{[15]}$. Sexual pleasure needs to be prioritized in the development of condoms and other sexual safety measures as well as in the promotion of their use ${ }^{[5]}$.

Seventy-two percent of our participants thought that their sexual life would be better without condom use; the reason that made women reject male condoms was to have bettersexual contactsensation and to be physically comfortable. Physical enjoyment (which facilitates orgasm and maximizes sexual enjoyment) involves sufficient arousal, the desired amount of lubrication, and appealing smell and touch. Condom side effects, for example, vaginal pain and dryness can all quell physical pleasure in agreement with other researchers ${ }^{[16,17]}$ who found that a greater proportion of their studied women disliked the feeling of male condoms and some women reported that condoms 'cover up' sensation and exacerbate vaginal dryness. Also, Bjekićc ${ }^{[18]}$ results indicated that the reason for not using a condom was that condom decreased sexual pleasure.

Klusmann ${ }^{[19]}$ concluded that an increase in the ability to achieve orgasm was associated with higher satisfaction scores. With condom use, orgasm was almost never reached in more than a quarter of our participants $(28 \%)$ and decreased enjoyment in $48 \%$ of them.Studies have shown that greater satisfaction with a relationship overall was associated with greater sexual satisfaction and fewer sexual function problems ${ }^{[13]}$; the stronger the emotional intimacy with the partner, the less the sexual distress ${ }^{[20]}$.

The current study showed that when condomswere used for other purposes other than contraception such as oral 
sex $(10 \%)$ or increasing sexual excitation $(1.5 \%)$, pleasing spouse or even pleasing both marital sides was the main goal. The Stone et al. ${ }^{[21]}$ study which explored oral sex and condom use among young people in the United Kingdom showed a high percentage of women (54\%) used condom for oral sex.

In our community, pleasing spouse is an important factor of sex appeal for women. For many women, satisfying men's physical and emotional needs maytake the form of abandoning male condom use. Sex goal is achieved when their husbands enjoy marital relationship. So, most of the women refuse condom after its trial and shift to condomless sex. For husbands of the current study, participant's unsatisfactory sex was the main complaint as reported by $64 \%$ of wives. This is in agreement withHiggins and Hirsch ${ }^{[16]}$ who found that women may reject the use of male condoms because they diminish men's pleasure, which may be a critical part of the women's own sexual enjoyment.

Loss of sensation, either real or imagined, is one of the main reasons that men prefer condomless sex. The common analogy is wearing a condom is like 'taking a shower with a raincoat on'[22].

On the other hand,condom use had some advantages in our study. Seventy-seven percent of women found that their husbands' use of condom increases coital duration. Moderate satisfaction of coitus duration with condom use was felt by $34 \%$ of these participants. Four percent of respondents used condom for treatment of premature ejaculation.Philpott et al. ${ }^{[23]}$ obtained similar results in India where condom promoters experienced longer lasting, and thus more pleasurable, intercourse.

Low arousal and low orgasm frequency in our participants were higher in rural areas, while satisfaction, surprisingly, was directly proportional to countryside participants. This may be due to feelings of embarrassment to declare that they are not satisfied with their sexual life even in a self-report questionnaire.

Among our participants, aged 20-39 years 27.7\% used a condom during oral sex. In an American study ${ }^{[24]}, 15.3 \%$ of women used a condom during the last oral sex. Cultural reasons may explain the difference as the American study found that Hispanic women were almost three times as likely as white women to report condom use at the last oral sex and married women were less likely to have used condoms at the last oral sex. We are not aware of the prevalence of oral sex in Egypt. On the other hand, women who had ever given oral sex during a lifetime in the United States amounted to $82.5 \%{ }^{[25]}$.

The ability of our participants to reach orgasm declined with advance in age. A previous report ${ }^{[26]}$ emphasized this finding. Egyptian old women undoubtedly, under societal pressure, find active sexual life incompatible with old age. Similarly, Basson ${ }^{[27]}$ suggests that older women may experience orgasm difficulty due to changes in their body and the belief that, at an older age, sexual desire and activity are improper. In contrast, Laumann et al. ${ }^{[28]}$ found that the frequency of orgasm both with a partner and during masturbation was greater for older groups of women and the lowest for women between 18 and 24 years of age. They attributed this to differences in the sexual experience such that younger women tend to have less experience and fewer partners.

The husbands of women aged 20-39 years always reported that they use condoms because they have no reliable contraceptive alternative. This is consistent with the results of Conley and Collins ${ }^{[29]}$ and Randolph et al. ${ }^{[30]}$ who found that men reported that condoms interfered with pleasure.

\section{LIMITATIONS OF THE STUDY}

People in Egypt are not used to talk about their sexual lives; many taboos persist in our population. Another limitation of this study is thatdata about husbands were reported by wives and we did not confirm the information with their husbands.

\section{CONCLUSION}

A majority of women disliked male condoms. Negative views associated with condoms are male in origin and internalized by women. Unsatisfactory sex, slipped condom, vaginal dryness, and pain were very common sexual side effects of condom use in our study.

\section{CONFLICT OF INTEREST}

There are no conflicts of interest.

\section{REFERENCES}

1. BancroftJ, GrahamCA, RamosR, MalayaC, FarleyTMM. The effects of steroidal contraceptives on the well-being and sexuality of women. A double-blind, placebo-controlled, twocenter study of combined and progesterone-only methods. Contraception1995; 52:363-369.

2. BrüllP, KesselsLTE, RepettoL, DirksonA, RuiterRAC. ERPs reveal disengagement processes related to condom use embarrassment in intentionbehavior inconsistent young adults. Arch Sex Behav2019; 48:521-532.

3. Helweg-LarsenM, CollinsBE. The UCLA Multidimensional Condom Attitudes Scale: documenting the complex determinants of condom use. Health Psychol1994; 13:224-237.

4. CataniaJA, DolciniMM, CoatesTJ, KegelesSM, GreenblattRM, PuckettS, et al. Predictors of condom use and multiple partnered sex among 
sexually-active adolescent women: implications for AIDS-related health interventions. J Sex Res1989; 26:514-524.

5. HenselDJ, StupianskyNW, HerbenickD, DodgeB, ReeceM. Sexual pleasure during condomprotected vaginal sex among heterosexual men. J Sex Med2012; 9:1272-1276.

6. CalabreseSK, ReisenCA, ZeaMC, PoppenPJ, BianchiFT. The pleasure principle: the effect of perceived pleasure loss associated with condoms. AIDS Patient Care STDs2012; 26:430-435.

7. MillerDJ, VachonDD, AalsmaMC. Negative affect and emotion dysregulation: conditional relations with violence and risky sexual behavior in a sample of justice-involved adolescents. Crim Justice Behav2012; 39:1316-1327.

8. RosenC, BrownJ, HeimanS, LeiblumC, MestonR, ShabsighD, et al.The Female Sexual Function Index (FSFI): a multidimensional self-report instrument for the assessment of female sexual function. J Sex Marital Ther2000; 26:191-208.

9. SchroderKE, JohnsonCJ, WiebeJS. An event-level analysis of condom use as a function of mood, alcohol use, and safer sex negotiations. Arch Sex Behav2009; 38:283-289.

10. Van den BrinkMJ, BoersmaAA, Meyboomde JongB, de BruijnJGM. Attitude toward contraception and abortion among Curaçao women. Ineffective contraception due to limited sexual education. BMC Fam Pract2011; 23:12-55.

11. WiegelM, MestonC, RosenR. The female sexual function index FSFI: cross-validation and development of clinical cutoff scores. J Sex Marital Ther2005; 31:1-20.

12. TschudinS, Meier-BurgoaJ, ArmbrusterU, SchwendkeA. Effects on the quality of life of a new oral contraceptive containing $30 \mathrm{mcg}$ EE and $3 \mathrm{mg}$ drospirenone (Yasmin). Praxis2003; 92:1177-1184.

13. LiRH, LoSS, TehDK, CheungKB, ChungTK. Impact of common contraceptive methods on quality of life and sexual function in Hong Kong Chinese women. Contraception 2004; 70:474-482.

14. WittingK, SanttilaP, AlankoK, AlgarsM, SandnabbaNK. Female sexual function and its associations with number of children, pregnancy and relationship satisfaction. J Sex Marital Ther2008; 34:89-106.
15. NewbyKV, BrownKE, FrenchDP, WallaceLM Which outcome expectancies are important in determining young adults' intentions to use condoms with casual sexual partners? a cross sectional study. BMC Public Health 2013; 13:133-141

16. HigginsJA, HirschJS. Pleasure and power: incorporating sexuality, agency, and inequality into research on contraceptive use and unintended pregnancy. Am J Public Health 2008; 98:1803-1813.

17. AmoryJK. Male contraception. Fertil Steril 2016; 106:1303-1309.

18. BjekićM, Sipetić-GrujičićS, VlajinacH, NikolićA. Reasons for not using condoms among heterosexual men in Belgrade, Serbia. Acta Dermatovenerol Croat2018; 26:58-60.

19. KlusmannD. Sexual motivation and the duration of partnership. Arch Sex Behav2002; 31:275-287.

20. BancroftJ, LoftusJ, LongJS. Distress about sex: a national survey of women in heterosexual relationships. Arch Sex Behav2003; 32:193-208.

21. StoneN, HatherallB, InghamR, McEachranJ. Oral sex and condom use among young people in the United Kingdom. Perspect Sex Reprod Health2006; 38:6-12.

22. SowPS, WardS. Continuing the Condom Conversation. Impatient Optimists, Bill and Melinda Gates Foundation. Available at:www.impatientoptimists.org/Posts/2013/03/ Reinventing-The-Condom [ Accessed October 4, 2018].

23. PhilpottA, KnerrW, MaherD. Promoting protection and pleasure: amplifying the effectiveness of barriers against sexually transmitted infections and pregnancy. Lancet2006; 368:2028-2031.

24. LeichliterJS, ChandraA, LiddonN, FentonKA, AralSO. Prevalence and correlates of heterosexual anal and oral sex in adolescents and adults in the United States. J Infect Dis2007; 196:1852-1859.

25. YounisI, El-EsawyF, Abdel-MohsenR. Is female orgasm an earth-moving experience: an Egyptian experience. Hum Androl2015; 5:37-44.

26. HayesR, DennersteinL. The impact of aging on sexual function and sexual dysfunction in women: a review of population-based studies. J Sex Med2005; 2:317-330.

27. BassonR. Women's sexuality and sexual 
dysfunction. In:GibbsRS, Karlan BY; Haney AF; Nygaard IE ed. Danforth's obstetrics and gynecology. 10th ed.Philadelphia, PA: Lippincott Williams and Wilkins; 2008. 742.

28. LaumannEO, PaikA, RosenRC. Sexual dysfunction in the United States: prevalence and predictors. JAMA1999; 281:537-544.
29. ConleyTD, CollinsBE. Difference between condom users and condom nonusers in their multidimensional condom attitudes. J App Soc Psychol2005; 35:603-620.

30. RandolphME, PinkertonSD, BogartLM, CecilH, AbramsonPR. Sexual pleasure and condom use. Arch Sex Behav2007; 36:844-848. 